\title{
Komplikationsmanagement bei vaskulären Interventionen
}

\author{
Thomas Jahnke, Stefan Müller-Hülsbeck
}

\section{Übersicht}

Einleitung

Definition und Einteilung

von Komplikationen

Vermeiden von Komplikationen

Fallbeispiele

\section{Einleitung}

Seit der ersten erfolgreichen Angioplastie durch Charles Dotter vor über 50 Jahren hat sich für eine Vielzahl vaskulärer Erkrankungen die interventionelle Radiologie als minimalinvasives Standardverfahren durchgesetzt. Interventionelle Prozeduren verlaufen in der überwältigenden Mehrheit der Fälle komplikationslos und zeichnen sich durch sehr hohe technische und klinische Erfolgsraten aus. Dennoch treten bei einem geringen Prozentsatz der Eingriffe Komplikationen auf, und jeder Arzt, der aktiv interventionelle Behandlungen durchführt, wird irgendwann im Rahmen seiner Tätigkeit mit teilweise schwerwiegenden unerwünschten Ereignissen konfrontiert.

Auch wenn der Schweregrad von Komplikationen mit der Komplexität der Prozeduren steigen kann, sind Zwischenfälle nicht selten unabhängig vom Ausbildungsstand des Interventionalisten. Auch der erfahrene Gefäßmediziner muss mit dem Auftreten von Komplikationen rechnen und Strategien zu deren Management parat haben, um Schaden vom Patienten abzuwenden. Thomas Edison hat einmal gesagt: „Erfahrung ist lediglich die Summe all unserer Fehler.“ Mit Einschränkungen trifft dies auch auf medizinische Eingriffe zu. Nur sind es in der Medizin idealerweise nicht die eigenen Fehler, die zu einer Verbesserung der Behandlungsqualität beitragen.
Die lebendigen und emotional geführten Diskussionen bei sog. M\&M-Konferenzen (Morbiditäts- und Mortalitätskonferenzen) zeigen, dass es in vielen Fällen nur der guten Idee eines Kollegen bedurft hätte, um ein akutes Problem zu lösen. Für die erfolgreiche Behandlung einer Komplikation kann es entscheidend sein, ob man von einem ähnlichen Problem bereits gehört hat. Durch die Präsentation von Fallbeispielen aus der klinischen Praxis wird die Möglichkeit geschaffen, direkt von der Erfahrung von Kollegen zu profitieren und diese für die Behandlung der eigenen Patienten zu nutzen.

Die in dem vorliegenden Beitrag vorgestellten Fallbeispiele stellen eine kleine Auswahl möglicher, etwas häufiger zu beobachtender Komplikationen bei vaskulären Interventionen dar und sollen Optionen zum Management der unerwünschten Ereignisse aufzeigen.

Es ist wichtig zu betonen, dass die in den folgenden Fallbeispielen letztlich durchgeführte Maßnahme nicht den Anspruch erhebt, die ideale Lösung eines spezifischen Behandlungsproblems zu sein.

Es ist schwierig, die große Palette an interventionellen Möglichkeiten in wenigen Fällen zu beleuchten. Dennoch geben die vorliegenden Beispiele einen praxisnahen Einblick in Möglichkeiten des interventionellen Komplikationsmanagements. 


\section{Definition und Einteilung von Komplikationen}

Das Auftreten von Komplikationen bei vaskulären Eingriffen wird in der Literatur mit Häufigkeiten zwischen 0 und $25 \%$ angegeben. Dabei ist die große Bandbreite der Literaturangaben das Resultat oft sehr unterschiedlicher Definitionen. Durchgesetzt hat sich bis heute die Einteilung von Komplikationen in sog. Minorund Majorkomplikationen:

- Minorkomplikationen: Darunter sind unerwünschte Ereignisse zu verstehen, die entweder keine klinische Konsequenz haben und auch keine weitere Therapie erfordern oder durch eine zusätzliche (interventionelle) Behandlung therapiert werden können und mit oder ohne Notwendigkeit einer stationären Überwachung über Nacht einhergehen.

- Majorkomplikationen: Diese führen immer zur Notwendigkeit einer intensivierten Therapie (interventionell oder medikamentös) oder einer Operation und verlängern den Krankenhausaufenthalt auf weniger oder auf mehr als $48 \mathrm{~h}$, gehen mit bleibenden Schäden einher oder führen zum Tode.

Verschiedene Faktoren können die Häufigkeit und Schwere von Komplikationen beeinflussen. Sie hängen mit der Erfahrung des Behandlers, dem Zustand des Patienten einschließlich der Komorbiditäten und der Art der zugrunde liegenden Läsion zusammen. Komplikationen können in jeder Phase eines interventionellen Eingriffs auftreten. Systemische Komplikationen

\section{Tabelle 1}

Einteilung von Komplikationen und unerwünschten Ereignissen im Rahmen von vaskulären Eingriffen nach ihrer Lokalisation nach Zeitler [1].

\begin{tabular}{|l|l|}
\hline Lokalisation & Komplikationen und unerwünschte Ereignisse \\
\hline Punktionsort & $\begin{array}{l}\text { Blutung, Hämatom, Aneurysma spurium, arteriovenöse Fistel, } \\
\text { arterieller Verschluss, venöse Thrombose, Komplikationen } \\
\text { durch Verschlusssysteme }\end{array}$ \\
\hline Behandlungsort & $\begin{array}{l}\text { Dissektion, Verschluss, Perforation, arteriovenöse Fistel, } \\
\text { Fehler des Katheter- bzw. des Stent-Materials oder uner- } \\
\text { wünschte Interaktionen zwischen Fremdmaterialien }\end{array}$ \\
\hline distale & $\begin{array}{l}\text { Embolie, Materialverschleppung, Aneurysma spurium, } \\
\text { Gefäß- und/oder Organverletzung, Non-Target-Embolisation }\end{array}$ \\
\hline systemisch & $\begin{array}{l}\text { kontrastmittelassoziiert (allergische Reaktion, renale Toxi- } \\
\text { zität, Schilddrüsenüberfunktion), Myokardinfarkt, Apoplex, } \\
\text { Postimplantationssyndrom, Postembolisationssyndrom, } \\
\text { Infektion }\end{array}$ \\
\hline
\end{tabular}

wie Myokardinfarkt oder Schlaganfall sind oft unabhängig von der eigentlichen Prozedur. Blutungen, Pseudoaneurysmabildung und arteriovenöse Fisteln stellen typische Zugangskomplikationen dar und Ereignisse wie Embolisation, Perforation und Dissektion hängen direkt mit dem Eingriff zusammen.

Nach ihrem Auftreten im Bereich der Punktionsstelle, im Bereich der eigentlichen Behandlungsregion oder der peripheren Strombahn lassen sich Komplikationen und systemische unerwünschte Ereignisse wie in Tab. 1 dargestellt einteilen.

\section{Vermeiden von Komplikationen}

\section{Vorbereitung des Eingriffs}

Entscheidend für die Vermeidung von Komplikationen ist die Vorbereitung des Eingriffs. So kann es im Rahmen von interventionellen Prozeduren zu Verwechslungen kommen. Mögliche Szenarien:

- Behandlung der falschen Läsion

- Durchführen einer inadäquaten Prozedur

- Behandlung der falschen Seite

- Behandlung des falschen Patienten

Diese Formen der Komplikation sind zwar selten, haben aber unter Umständen katastrophale Auswirkungen sowohl in medizinischer als auch in medikolegaler Hinsicht.

Verwechslungskomplikationen lassen sich aber zuverlässig vermeiden. Die Implementierung von SOP (Standard Operating Procedures) hilft, die Gefahr einer Verwechslung durch die strikte Einhaltung folgender 3 Prozesse während der Interventionsvorbereitung zu minimieren:

- präinterventionelle Patientenverifizierung

- Markierung des Interventions- bzw. des Zugangsorts

- sog. Team-Time-out unmittelbar vor Eingriffsbeginn

Es ist bei der Interventionsvorbereitung wichtig, dass alle Mitglieder des prä- und perioperativen Teams aktiv in diesen Prozess eingebunden sind und miteinander kommunizieren. Des Weiteren ist der Patient, soweit möglich, miteinzubinden.

Die Implementierung von Checklisten und Arbeitsanweisungen zum Umgang mit z. B. renaler Insuffizienz, latenter Hyperthyreose und allergischer Diathese hilft zusätzlich, Komplikationen zu vermeiden [2,3]. 


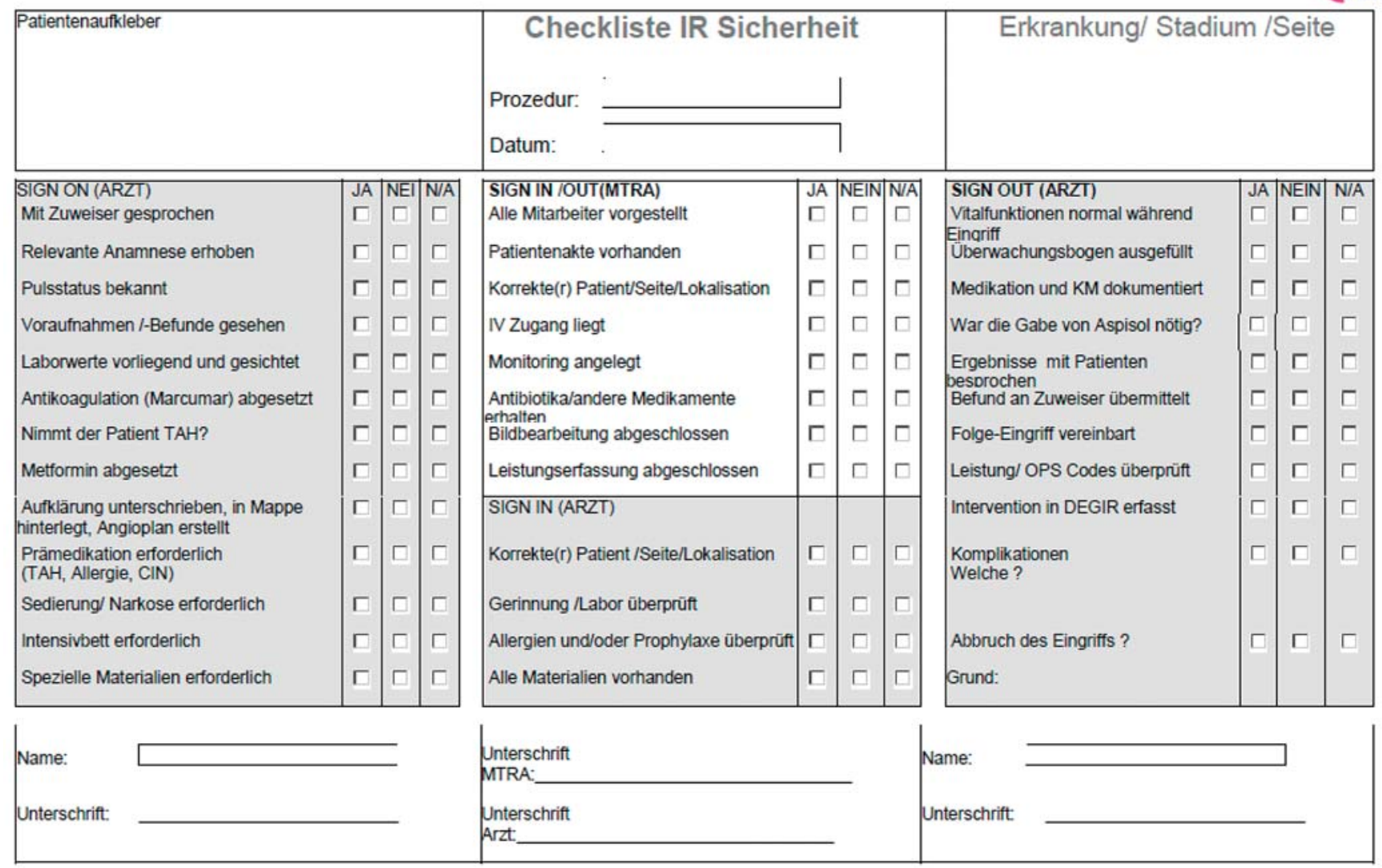

Abb. 1 JR-Checkliste in Anlehnung an die CIRSE IR Patient Safety Checklist [4]. CIN= Kontrastmittel induzierte Nephropathie; DEGIR=Q Qualitätssicherungsregister der Deutschen Gesellschaft für Interventionelle Radiologie und minimalinvasive Therapie IR = interventionelle Radiologie; IV=intravenös; KM = Kontrastmittel; OPS = Operationen- und Prozedurenschlüssel; TAH=Thrombozytenaggregationshemmer

Die Autoren empfehlen die Patientenvorbereitung gemäß der Sicherheitscheckliste der CIRSE IR Patient Safety Checklist der CIRSE (Cardiovascular and Interventional Radiological Society of Europe) [4,5]. Sie enthält wichtige Punkte, die Kommunikationsproblemen und Informationsdefiziten vorbeugen helfen. Die CIRSE-Checkliste kann an die eigenen Prozesse angepasst und entsprechend modifiziert werden (Abb.1).

\section{Periprozedurale Dokumentation}

Es wird empfohlen, alle Schritte einer Intervention im Befund zu dokumentieren und Bilder und/oder Serien des Eingriffs umfassend zu archivieren [6, 7].

Dies gilt für Eingriffe ohne Vorkommnisse genauso wie für Fälle mit unerwünschten Ereignissen. Zur Komplikationsvermeidung gehört es, sich vor Beginn einer Prozedur mit allen vorhandenen Voraufnahmen vertraut zu machen. Auch ist eine umfassende Dokumentation des Interventionsverlaufs von unschätzbarem
Wert für evtl. erforderliche Folgeeingriffe und hat nicht zuletzt wichtige Bedeutung für die Abwendung möglicher Haftungsansprüche.

\section{Fallbeispiele}

\section{Fall 1: Interaktion zwischen einem hydrophil beschichteten Draht und einer Seldinger-Punktionsnadel}

\section{Anamnese}

Ein 60 Jahre alter Patient wurde aufgrund einer wiederaufgetretenen PAVK (periphere arterielle Verschlusskrankheit) für eine diagnostische Angiografie vorgesehen. Während der retrograden Punktion gab es Probleme bei der Einlage eines atraumatischen 0,035Inch-Schleusendrahts über die Seldinger-Punktionsnadel. Daraufhin wurde ein hydrophil beschichteter 0,035-Inch-Draht verwendet. Dieser wurde in die A. iliaca externa eingebracht. Der Draht wurde gegen einen Widerstand durch die Nadel zurückgezogen. 
Dabei kam es zu einem partiellen Abschälen der hydrophilen Beschichtung, und Teile dieser hydrophilen Beschichtung verblieben in der A. femoralis communis.

\section{Initiale Behandlung}

Gescheiterter retrograder Leistenzugang.

\section{- Aufgetretenes Problem während der Behandlung}

Die retrograde Platzierung eines atraumatischen Schleusendrahts scheiterte. Daraufhin wurden Versuche unternommen, einen hydrophil beschichteten Führungsdraht zur sicheren Schleusenplatzierung an Ort und Stelle zu bringen. Aus unerklärlichen Gründen wurde dieser Draht partiell über die Seldinger-Nadel zurückgezogen. Dabei kam es zum Abschälen von hydrophiler Beschichtung und zum Verbleib dieser Beschichtung im Gefäß (Abb. 2a).

\section{- Plan für die Bildgebung}

Angiografie über die kontralaterale Leiste.

\section{Komplikation}

Fremdkörper in der A. femoralis communis.

- Mögliche Strategien zur Komplikationsbehandlung

- Endovaskuläre Behandlung über einen kontralateralen Leistenzugang

- Platzierung einer Schleuse zum Einfangen des Fremdkörpers

- chirurgische Leistenfreilegung, wenn der endovaskuläre Versuch scheitert

\section{Durchgeführte Behandlung der Komplikation} Kontralaterale Leistenpunktion und Einlage einer $45 \mathrm{~cm}$ messenden 6-F-Schleuse, die in Cross-overTechnik platziert wurde. Mittels Schlinge konnte schließlich der Fremdkörper extrahiert werden (Abb.2b und Abb.2c).

\section{- Komplikationsanalyse}

Eine unkontrollierte Manipulation eines hydrophil beschichteten Drahtes in Kombination mit einer scharfen Seldinger-Nadel kann zur oben beschriebenen Komplikation mit Abschälung der hydrophilen Drahtbeschichtung führen.

\section{Strategien zur Prävention}

- Es sollte niemals ein Draht unkontrolliert durch eine Seldinger-Nadel zurückgezogen werden.

- Sollte es während des Zurückziehens zu einem Widerstand kommen, dann muss der Draht einschließlich Punktionsnadel entfernt werden.

- Im Idealfall gelingt es durch ein Schlingenmanöver, den abgescherten Fremdkörper wieder zu entfernen.

\section{Weiterführende Literatur}

Bezüglich weiterer Informationen zu dieser Komplikation und ihrer Prävention s. [8-13].
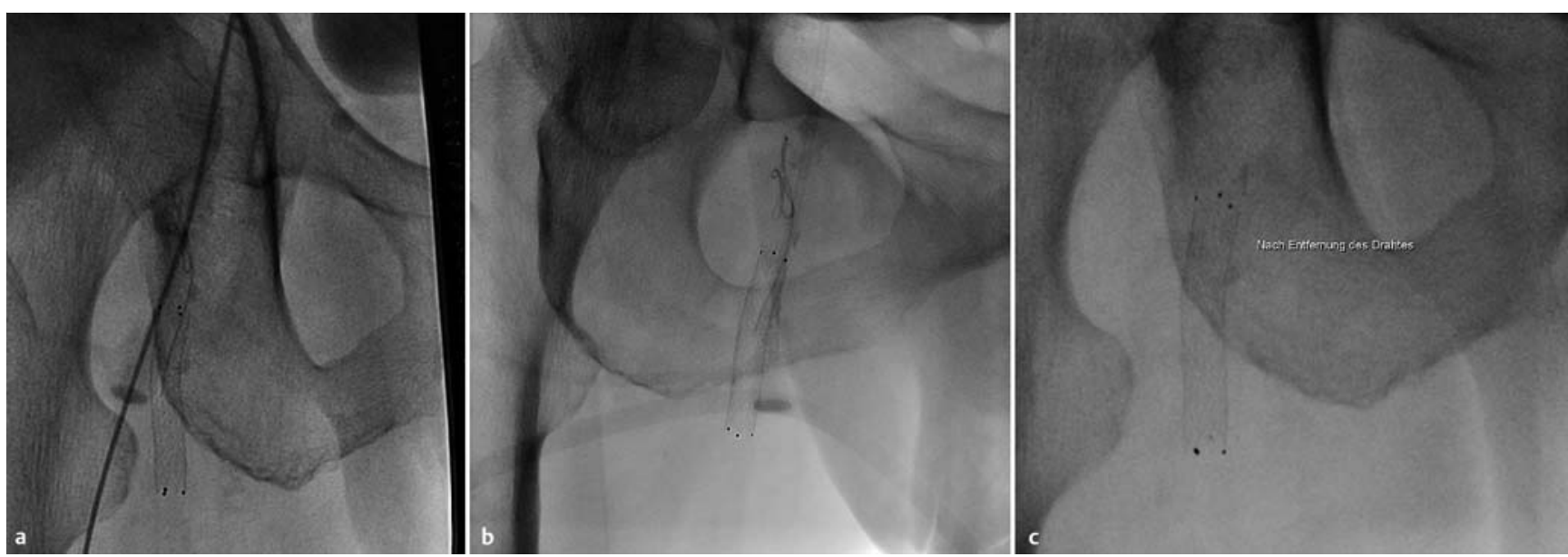

Abb.2 Abgescherte hydrophile Drahtbeschichtung auf Höhe des Abgangs der A. femoralis superficialis. a Abgeschälte hydrophile Beschichtung. Ein selbstexpandierender Stent ist in der proximalen A. femoralis superficialis implantiert. b Eine in Cross-over-Technik platzierte Schlinge kann Teile des Führungsdrahts fassen. c Durchleuchtungsbild nach vollständiger Entfernung des Drahtrests. 

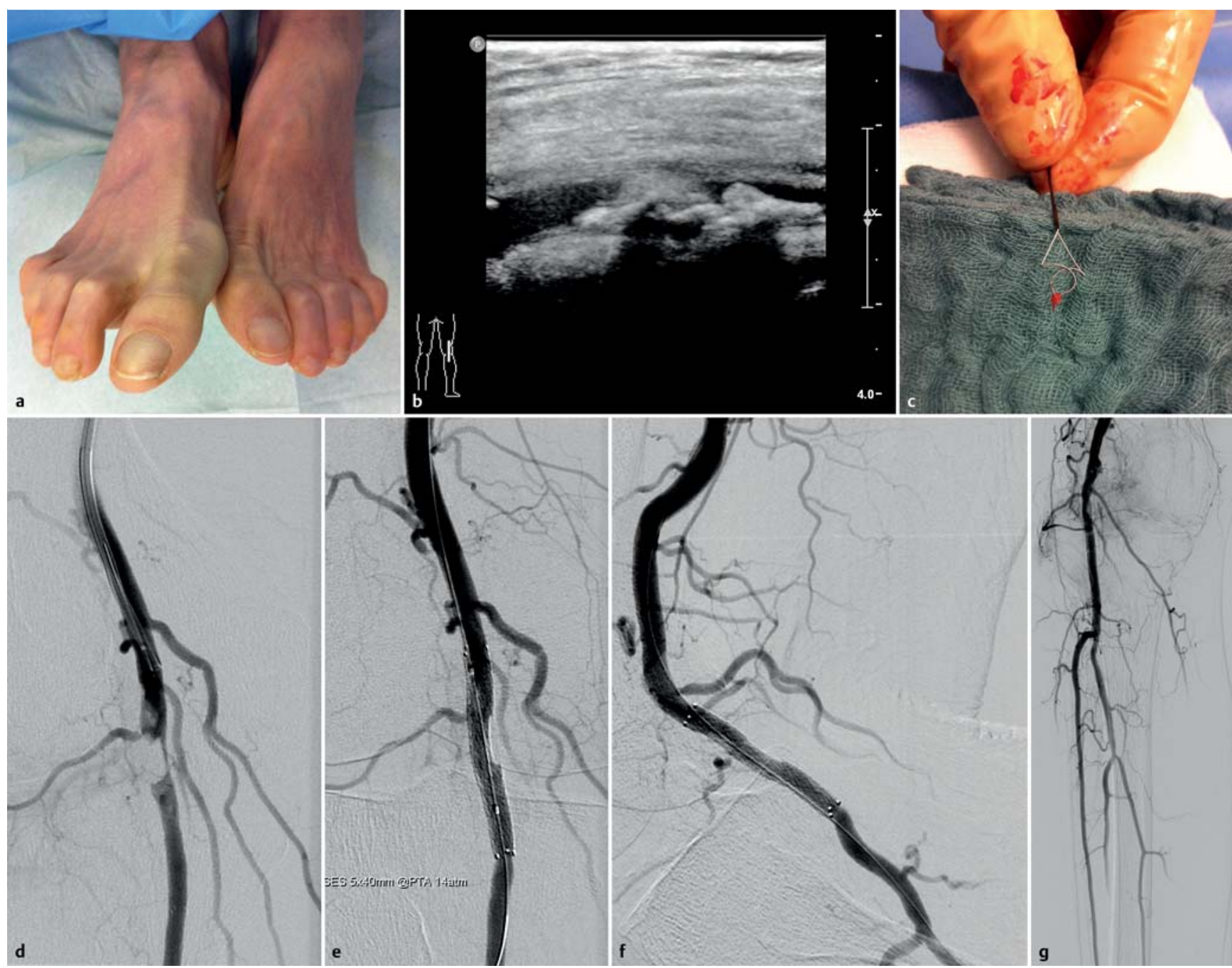

Abb.3 Embolisation eines Exoseal-Kollagenplaques in die distale A. femoralis superficialis nach retrogradem Gefäßverschluss. a Abgeblasster rechtsseitiger Vorfuß.b B-Mode-Ultraschallbild, das den Kollagenplaque im Vessellumen an einen exzentrischen Intravaskularplaque angeheftet zeigt. c Vergrößerungsaufnahme der Schlinge mit geringen Kollagenresten an der Schlinge. $\mathbf{d}$ Kontrollangiografie nach mehreren Schlingenmanövern. Sie zeigt immer noch eine signifikante, hämodynamisch relevante Reststenose. e Kontrollangiografie im a.-p. (anteroposterioren) Strahlengang nach Kniebeugung nach Implantation eines selbstexpandierenden Stent. PTA = perkutane transluminale Angioplastie; SES = selbstexpandierender Stent; $\mathbf{f}$ Korrespondierende Kontrollangiografie im seitlichen Strahlengang. g Abschließende Kontrollangiografie des Unterschenkels ohne Anhaltspunkte für das Vorliegen distaler Embolien.

Fall 2: Embolisation eines Exoseal-Plaques in die distale Arteria femoralis superficialis nach retrogradem Gefäßverschluss

\section{Anamnese}

Ein 70-jähriger Patient erhielt eine Woche zuvor eine Koronarangiografie; der Leistenverschluss war erfolgreich. Die Wiederaufnahme in die Klinik beruhte auf einer nun eingeschränkten Gehstrecke von $20 \mathrm{~m}$, die vor der Koronarangiografie nicht bestand. Aktuell lag auch eine Abblassung des Vorfußes vor (Abb.3a).

\section{Initiale Behandlung}

Unkomplizierte Koronarangiografie mit retrogradem

Gefäßverschluss unter Verwendung von Exoseal 6 F.

\section{- Aufgetretenes Problem während der Behandlung}

Das Gefäßverschlusssystem wurde entsprechend den Herstellerangaben verwendet. Nach Aktivierung des Systems konnte keine Hämostase erreicht werden. Deshalb wurde eine manuelle Kompression für weitere 5 min bis zum Erreichen der Hämostase durchgeführt.

\section{- Plan für die Bildgebung}

Farbcodierte Duplexsonografie der Leiste und der ipsilateralen infrainguinalen Beinstrombahn. 


\section{- Komplikation}

Embolisation eines Exoseal-Kollagenplaques in die distale A. femoralis superficialis mit daraus resultierender hochgradiger Stenose (Abb. 3b).

\section{- Mögliche Strategien zur Komplikations- behandlung}

- Endovaskuläre Behandlung mittels Stent-Implantation.

- ipsilaterale antegrade Punktion zur endovaskulären Entfernung durch Schlingen- oder Aspirationsmanöver

- offen-chirurgische Freilegung

- Atherektomie mit Filterprotektion

\section{- Durchgeführte Behandlung der Komplikation}

Endovaskuläre Behandlung durch Stent-Fixierung des Kollagenplaques. Versuche der Aspiration oder eines Schlingenmanövers zum Entfernen des Plaques scheiterten. Lediglich geringe Anteile des Verschlussmaterials konnten entfernt werden (Abb.3c). Eine Angiografie über eine antegrad eingebrachte Schleuse zeigte das exzentrisch gelegene und stenosierende Verschlussmaterial (Abb. 3d). Schließlich wurde eine Implantation eines selbstexpandierenden Stent $(5 \times 40 \mathrm{~mm}) \mathrm{mit}$ nachfolgender PTA (perkutane transluminale Angioplastie) durchgeführt (Abb.3e und Abb. 3f). Die angiografische Abschlusskontrolle zeigte einen prompten Kontrastmittelabstrom (Abb.3g).

\section{- Komplikationsanalyse}

Die Ursache für diese Komplikation bleibt unklar. Wahrscheinlich kam es zu einer falschen Aktivierung des Exoseal-Systems, die zu einer Embolie des Verschlussstopfens in die distale A. femoralis superficialis führte.

\section{Strategien zur Prävention}

- Vor Anwendung eines Gefäßverschlusssystems ist das Gefäßsegment angiografisch auf seine Eignung zu überprüfen. Die Herstellerangaben zur Anwendung des Verschlusssystems müssen bekannt sein.

- Bei Unklarheiten sollte im Zweifelsfall das Verschlusssystem entfernt und eine manuelle Kompression durchgeführt werden.

\section{Weiterführende Literatur}

Bezüglich weiterer Informationen zu dieser Komplikation und ihrer Prävention s. [14-20].

\section{Fall 3: Seitenastperforation durch einen hydrophil beschichteten Draht}

\section{Anamnese}

Ein 68-jähriger Patient mit pAVK-Symptomatik stellte sich zur Behandlung einer hochgradigen Stenose der A. femoralis superficialis vor. Die Stenosebehandlung erfolgte nach antegrader Punktion. Ein hydrophil beschichteter 0,018-Inch-Draht wurde in das Gefäß eingelegt. Ein selbstexpandierender Stent wurde aufgrund einer flussrelevanten Dissektion zur Stenosebehandlung implantiert. Zahlreiche PTA-Katheter mussten eingeführt werden, um das Ergebnis zu komplettieren. Während der gesamten Prozedur wurde der 0,018-Inch-Führungsdraht nicht ausgetauscht. Hervorzuheben ist jedoch, dass der Patient während der Intervention kurzfristig ein heftiges Schmerzereignis angab, das allerdings selbstlimitierend war; es dauerte nur wenige Sekunden an. Aufgrund des Schmerzereignisses wurde eine Durchleuchtung des Unterschenkels durchgeführt und nach Zurückziehen des Führungsdrahts eine ergänzende Kontrollangiografie, die einen Kontrastmittelaustritt in 2 verschiedenen infragenualen Seitästen zeigte (Abb.4a).

\section{- Initiale Behandlung}

PTA und Stent-Implantation zur Behandlung einer pAVK.

\section{- Aufgetretene Probleme während der Behandlung}

Selbstlimitierendes, vom Patienten angegebenes Schmerzereignis; ansonsten unkomplizierter Interventionsablauf. Während der Kontrollangiografie bemerkte Blutung aus infragenualen Seitästen.

\section{- Plan für die Bildgebung}

Selektive Angiografie zur genaueren Lokalisation der Blutung.

\section{Komplikation}

Seitenastperforation.

\section{Mögliche Strategien zur Komplikations- behandlung}

- Manuelle Kompression des Unterschenkels für 3 min in Höhe der zu vermutenden Gefäßperforation

- Anlegen einer Blutdruckmanschette um den Unterschenkel, die für 3 min zwischen systolischem und diastolischem Blutdruck insuffliert wird (Fußpulse sollten tastbar bleiben); ggf. Wiederholen des Manövers bis zum Sistieren der Blutung 
- selektive Embolisation des zuführenden Gefäßes durch einen Mikrokatheter mittels Embolisation (Coil, Kleber, Kollagen)

\section{- Durchgeführte Behandlung der Komplikation}

Zunächst Insufflation einer Blutdruckmanschette für 3 min unterhalb des Knies. Wiederholung dieses Manövers, bis die Blutung angiografisch nicht mehr nachweisbar war. Kontrolle der Blutung durch Kontrollangiografie (Abb.4b).

\section{Komplikationsanalyse}

Ein nicht bemerktes, unkontrolliertes Vorschieben der Spitze eines hydrophil beschichteten Führungsdrahts führte zur 2-fachen Perforation eines Seitasts auf unterschiedlichen Ebenen.

\section{Strategien zur Prävention}

- Es sollte immer auf die distale Spitze des hydrophil beschichteten Führungsdrahts geachtet werden.

- Wichtig ist die visuelle Kontrolle der distalen Führungsdrahtspitze während des Vorschiebens von Kathetern oder anderen Interventionsmaterialien und bei deren Austausch.

- Hinweise des Patienten sind immer ernst zu nehmen. Nach Schmerzereignis muss die Führungsdrahtlage kontrolliert und ggf. eine Kontrollangiografie durchgeführt werden.

\section{Weiterführende Literatur}

Bezüglich weiterer Informationen zu dieser Komplikation und ihrer Prävention s. [21 -28].

\section{Fall 4: Stent-Migration während einer Kissing-Stent-Prozedur}

\section{Anamnese}

Es stellte sich ein 70-jähriger Patient mit pAVK-Symptomatik und hämodynamisch relevanter Stenose vor; diese Stenose involvierte die Aortenbifurkation. Für den Patienten wurde eine Kissing-Stent-Prozedur geplant (Abb.5a).

\section{- Initiale Behandlung}

Unter Lokalanästhesie retrograde Leistenpunktion beidseits und Einlage von 0,035-Inch-Drähten in die Aorta abdominalis. Geplante Stent-Implantation eines $8 \times 59 \mathrm{~mm}$ messenden ballonexpandierbaren Stent linksseitig in die A. iliaca communis und rechtsseitig Implantation eines $9 \times 29 \mathrm{~mm}$ messenden Stent in die A. iliaca communis.

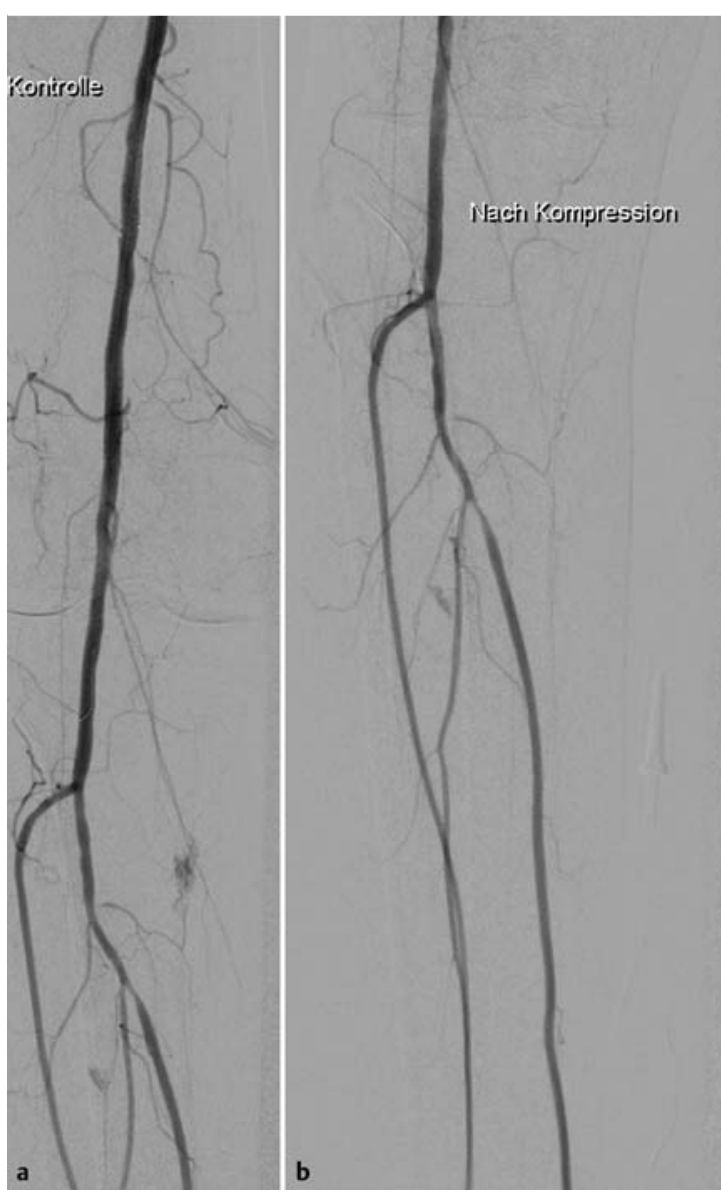

Abb. 4 Seitenastperforation durch einen hydrophil beschichteten Draht. a Digitale Subtraktionsangiografie, die eine Seitastperforation infragenualer Seitäste in 2 unterschiedlichen Ebenen aufzeigt. b Kontrollangiografie nach erfolgreicher Insufflation einer Blutdruckmanschette. Eine signifikante Gefäßperforation ist nicht mehr nachweisbar.

\section{- Aufgetretenes Problem während der Behandlung}

Der linksseitige Stent wurde ohne Probleme vorgeschoben und am Zielort positioniert, während der rechtsseitige Stent aus unerfindlichen Gründen vom Ballon migrierte. Der Operateur entfernte den Ballon und der Stent wurde vor der Schleuse gestoppt.

\section{- Plan für die Bildgebung}

Durchleuchtungsbild, Vergrößerungsaufnahme zur exakten Positionsbestimmung des Stent und zur Beurteilung möglicher Stent-Deformierungen (Abb.5b).

\section{Komplikation}

Stent-Migration und milde Stent-Deformierung.

\section{Mögliche Strategien zur Komplikations- behandlung}

- Endovaskuläre Behandlung durch Stent-Entfaltung in der A. iliaca externa durch einen Ballon mit geringem Durchmesser

- endovaskuläre Behandlung mit Versuch des Vorschiebens des Stent und Positionierung am Zielort durch einen eingeführten Ballonkatheter 

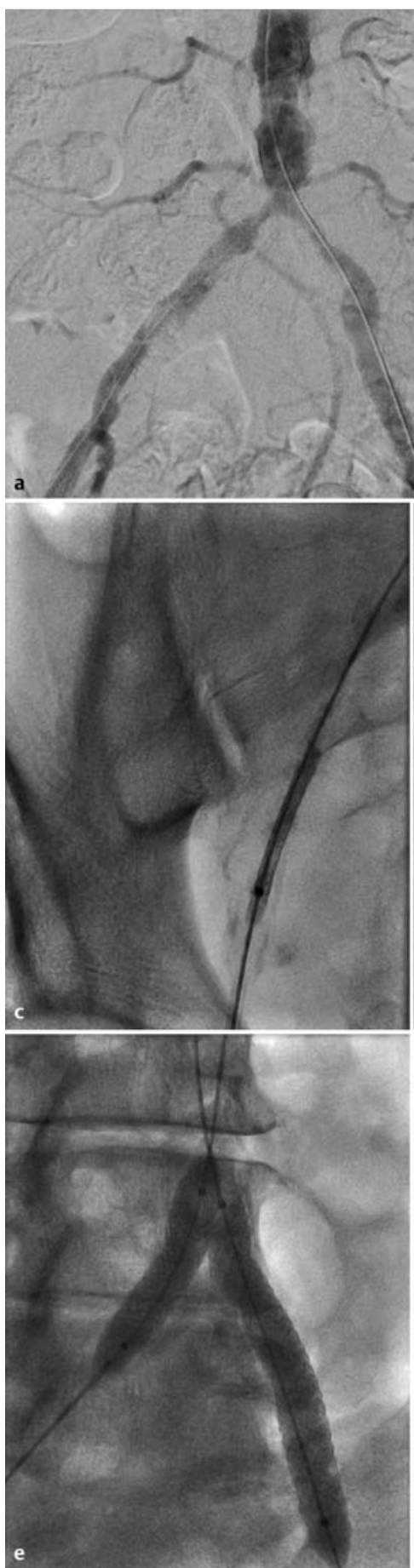

d

Abb. 5 Stent-Migration während einer Kissing-Stent-Prozedur. a Die diagnostische Angiografie zeigt eine relevante Stenose der A. iliaca communis beidseits und der distalen Aorta abdominalis. b Der partiell deformierte Stent stößt an die Schleuse an und ist vom Trägerkatheter migriert. c Mithilfe eines dünnen 4-mm-Ballons, der in den Stent eingeführt und partiell aufgedehnt worden ist, kann der Stent von der A. iliaca externa zur A. iliaca communis vorgeführt werden. $\mathbf{d}$ Dort kann er entfaltet werden. e Nach Einwechseln eines im Durchmesser $8 \mathrm{~mm}$ messenden Ballons kann die Kissing-Stent-Prozedur durch beidseitiges Aufdehnen der Ballons komplettiert werden.

- endovaskuläre Behandlung mit Schlingenmanöver und Versuch der Stent-Entfernung

- chirurgische Stent-Entfernung, wenn endovaskuläre Versuche versagen

\section{Durchgeführte Behandlung der Komplikation}

Endovaskuläre Behandlung: Ein $4 \times 80 \mathrm{~mm}$ messender Ballon konnte in den nicht entfalteten Stent eingeführt werden. Durch partielles Aufdehnen des Ballons (Abb. 5c und Abb.5d) konnte der Ballon mit dem Stent am Zielort positioniert und schließlich bis auf $4,5 \mathrm{~mm}$ aufgedehnt werden. Nach Auswechseln des Ballonkatheters wurde ein $8 \times 40 \mathrm{~mm}$ messender Ballonkatheter eingeführt und es wurde eine simultane Aufdehnung durchgeführt (Abb. 5e). Durch dieses Manöver konnte die Kissing-Stent-Prozedur komplettiert werden.

\section{Komplikationsanalyse}

Es kam vermutlich zu einem unkontrollierten Vorschieben eines Stent-Ballonkatheters mit daraus resultierender Stent-Migration.

\section{Strategien zur Prävention}

- Wie alle endovaskulären Instrumente sollten auch Stent-Ballonkatheter kontrolliert und vorsichtig unter Durchleuchtung vorgeschoben werden. Dies gilt insbesondere für ballonexpandierbare Stents.

- Kann ein ballonexpandierbarer Stent die Läsion nicht passieren, so ist eine vorsichtige Vordilatation erforderlich.

- Wenn ein Zurückziehen des Stent erforderlich ist, dann muss dieser mit größter Vorsicht in die Schleuse zurückgeführt werden, und zwar unter Durchleuchtungskontrolle. Ein Stent- bzw. Ballonkatheter sollte nie unkontrolliert und gegen einen Widerstand vor- oder zurückgeschoben werden.

- Der Führungsdraht darf niemals aufgegeben werden.

\section{Weiterführende Literatur}

Bezüglich weiterer Informationen zu dieser Komplikation und ihrer Prävention s. [29-31].

\section{Fall 5: Stent-Fehlplatzierung in einen poplitealen Seitast}

\section{- Anamnese}

Eine 58-jährige Patientin mit Wundheilungsstörungen am Fuß wurde für eine Rekanalisation der A. poplitea und der distalen A. femoralis superficialis vorgesehen. 

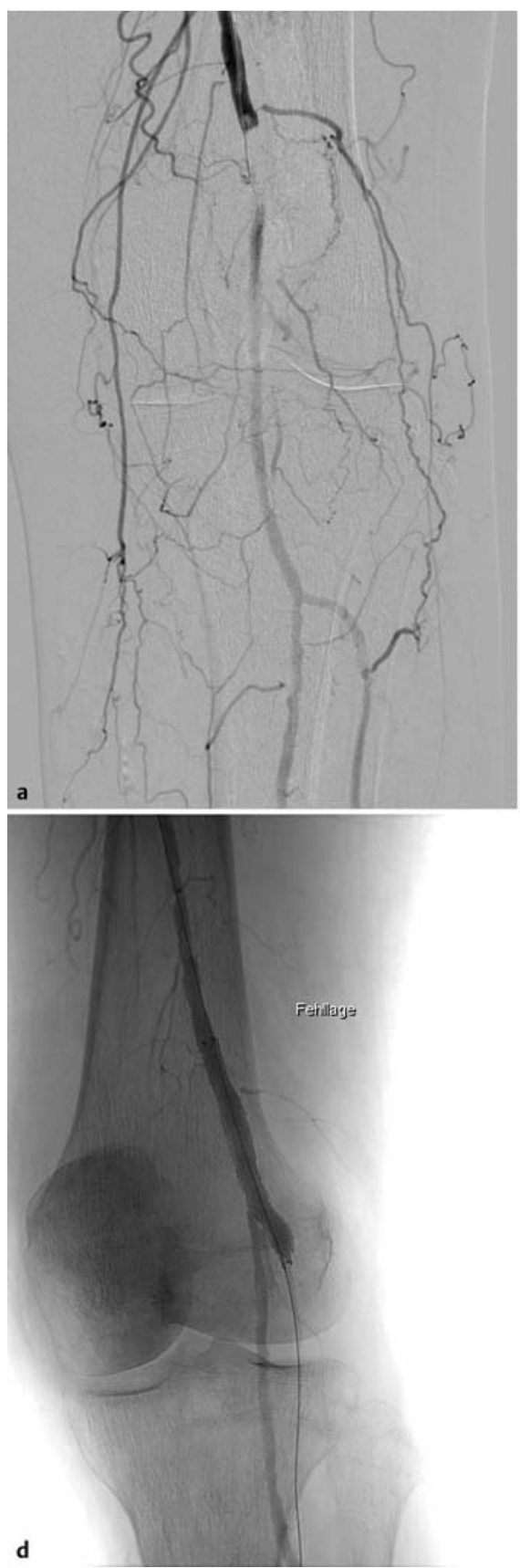

\section{- Initiale Behandlung}

Antegrade Punktion der linken A. femoralis communis und Einlage einer Terumo-6-F-Schleuse. Anschließend Rekanalisation des Verschlusses mittels hydrophil beschichtetem 0,018-Inch-Führungsdraht. Nach erfolgreicher Rekanalisation und PTA war das Segment am Übergang von der A. femoralis superficialis zur A. poplitea verschlossen (Abb.6a). Ein Abweichen des Führungsdrahts in einen Seitast wurde bemerkt und die Lage des Drahtes wurde korrigiert (Abb.6b). Ein selbstexpandierender Stent wurde implantiert $(6 \times 60 \mathrm{~mm})$. Die Kontrollangiografie zeigte ein erneutes

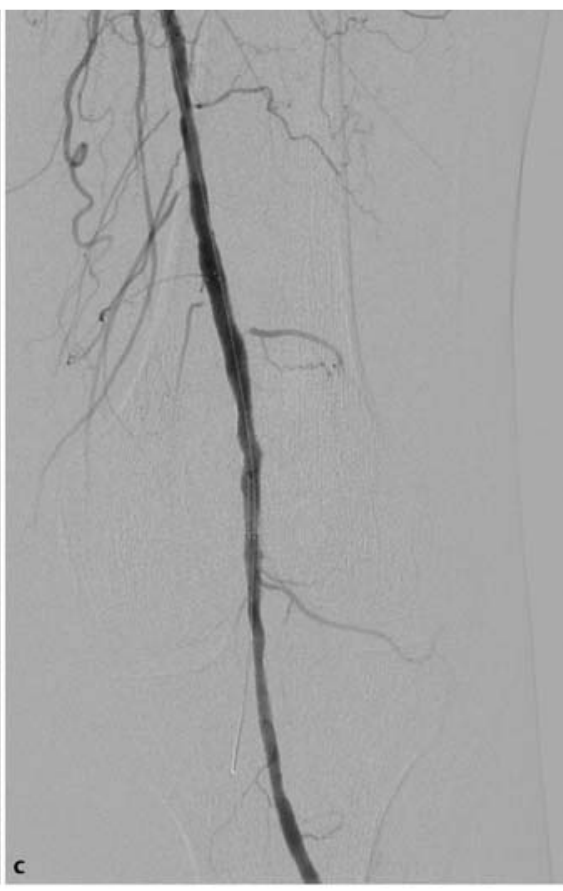

Abb. 6 Stent-Fehlplatzierung in einem poplitealen Seitast. a Diagnostische Angiografie nach Rekanalisation eines kurzstreckigen distalen A.-femoralis-superficialis- und P1-Verschlusses mit einem Führungsdraht.b Nach erfolgreicher PTA wird festgestellt, dass der Führungsdraht in einen Seitast abweicht. Die Lage wird entsprechend korrigiert. c Angiografie nach Stent-Implantation. Der distale Führungsdraht weicht infragenual in einen Seitast ab. $\mathbf{d}$ Schrägprojektion in angiografischer Darstellung: 2 parallel verlaufende Gefäße der A. poplitea und eines Seitasts, in dem das distale Ende des Stent platziert wurde, da im Seitast der Führungsdraht verläuft. e Nach sog. Crush-Stenting-Prozedur zeigt die finale Angiografie einen guten Kontrastmittelabstrom, nachdem der fehlplatzierte Stent mit einem weiteren selbstexpandierenden Stent penetriert und in die A. poplitea implantiert wurde. nicht vollständig distal entfaltet hatte. Eine Angiografie in angulierter Projektion zeigte den parallelen Verlauf zur A. poplitea. Das distale Stent-Ende wurde in einen parallel verlaufenden Seitast platziert.

\section{- Aufgetretenes Problem während der Behandlung}

Fehlplatzierung eines selbstexpandierenden Stent in einen poplitealen Seitast. 


\section{- Plan für die Bildgebung}

Diagnostische Angiografie und Fluoroskopie zur Bestimmung der exakten Stent-Lage.

\section{Komplikation}

Stent-Fehlplatzierung in einen poplitealen Seitast.

\section{- Mögliche Strategien zur Komplikationsbehandlung}

- Belassen des Ist-Zustands; Fortführen der dualen Plättchenhemmung für mindestens 8 Wochen oder länger

- ergänzende Implantation eines selbstexpandierenden Stent nach Repositionierung des Führungsdrahts in die distale A. poplitea und Durchführung eines sog. Crush-Stenting

\section{Durchgeführte Behandlung der Komplikation}

Implantation eines 2. selbstexpandierenden Stent (6× $30 \mathrm{~mm}$ ), nachdem der Führungsdraht zurückgezogen worden war, das offene Zelldesign des primär implantierten Stent passiert hatte und in der distalen A. poplitea platziert worden war (Abb.6d und Abb.6e).

\section{- Komplikationsanalyse}

Gebogene hydrophil beschichtete Drähte haben die Tendenz, in Seitäste zu wandern. Nicht immer werden die Position eines Seitasts und sein Abgang korrekt eingeschätzt. Im vorliegenden Fall erschien die Position des Führungsdrahts lediglich distal falsch. Es wurde während des gesamten Eingriffs im a.-p.(anteroposterioren) Strahlengang gearbeitet und nicht bemerkt, dass es zu einer Fehlplatzierung des Führungsdrahts gekommen war, da über eine gewisse Länge die A. poplitea und ein Seitast im a.-p.Strahlenast parallel und in einer Ebene verlaufen.

Um diese Komplikation zu vermeiden, ist es wichtig, im Zweifelsfall Schrägprojektionen durchzuführen, um die Führungsdrahtlage korrekt einschätzen zu können.

\section{Strategie zur Prävention}

Interventionell sind Instrumente mit größter Vorsicht zu benutzen. Die distale Position eines Führungsdrahts ist immer zu evaluieren. Gegebenenfalls muss die angiografische Ebene während einer Prozedur geändert werden, um den sicheren Verlauf eines Führungsdrahts einschätzen und evtl. korrigieren zu können.

\section{Weiterführende Literatur}

Bezüglich weiterer Informationen zu dieser Komplikation und ihrer Prävention s. [32,33].

\section{Fazit}

Interventionelle Eingriffe verlaufen in der großen Mehrheit der Fälle ohne Komplikationen und sehr erfolgreich. Dennoch erlebt auch der erfahrene Interventionalist hin und wieder das Auftreten von Komplikationen. Um im Bedarfsfall schnell und adäquat reagieren zu können, ist die Kenntnis möglicher Komplikationen unerlässlich.

Auf die Häufigkeit und Schwere von Komplikationen wirken sich ganz unterschiedliche Faktoren aus, so auf der einen Seite die Erfahrung des Behandlers und die Komplexität des Eingriffs und auf der anderen Seite der Allgemeinzustand des Patienten, seine Komorbiditäten und die Art der Läsion. Systemische Komplikationen können unabhängig vom durchgeführten Eingriff auftreten, andere Komplikationen hängen direkt mit dem Eingriff zusammen.

Durch eine durchdachte Vorbereitung des Patienten lässt sich bereits eine große Zahl von Komplikationen vermeiden. Die konsequente Durchführung von festgelegten SOP leistet dabei eine große Hilfestellung, ebenso die Bereitstellung von Checklisten und Arbeitsanweisungen für häufiger auftretende Komplikationen. Die umfassende Dokumentation des gesamten Eingriffs ist von gleicher Wichtigkeit. Anhand von Fallbeispielen von Kollegen kann sich der Interventionalist zudem über in der Praxis auftretende Komplikationen, ihre Ursachen sowie ihre Behandlung informieren. 


\section{Über die Autoren}

\section{Thomas Jahnke}

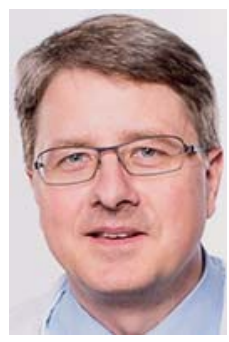

Prof. Dr. med.; 1989 - 1997 Studium der Humanmedizin an der Universität Kiel. 1999-2009 Klinik für Diagnostische Radiologie am Universitätsklinikum Schleswig-Holstein, Campus Kiel. 2005 Habilitation. 2007 Stellvertretender Direktor der Klinik. Seit 2010 Chefarzt der Klinik für Diagnostische und Interventionelle Radiologie/Nuklearmedizin am Friedrich-Ebert-Krankenhaus (FEK) Neumünster.

\section{Stefan Müller-Hülsbeck}

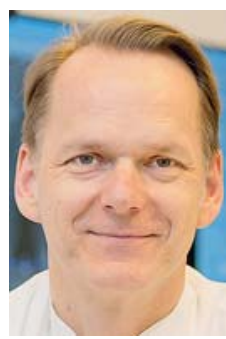

Prof. Dr. med.; 1984 - 1991 Studium der Humanmedizin an der Universität Kiel. 1993-2007 Klinik für Diagnostische Radiologie am Universitätsklinikum Schleswig-Holstein, Campus Kiel. 2000 Habilitation. 2003 Stellvertretender Direktor und Leitender Oberarzt der Klinik. Seit 2007 Chefarzt der Abteilung für diagnostische und interventionelle Radiologie/Neuroradiologie des medizinischen Klinikverbundes Flensburg, Ev.-Luth. Diakonissenanstalt zu Flensburg (Diako).

\section{Korrespondenzadresse}

Prof. Dr. med. Thomas Jahnke

Friedrich-Ebert-Krankenhaus GmbH

Klinik für Diagnostische und Interventionelle Radiologie/ Nuklearmedizin

Friesenstr. 11

24534 Neumünster

E-Mail: thomas.jahnke@fek.de

\section{Literatur}

1 Zetiler E. Complications in and after PTR. In: Zeitler E, Grüntzig A, Schoop W (eds.) Percutaneous vascular recanalization. Berlin: Springer; 1978: 120-150

2 Omary RA, Bettmann MA, Cardella JF et al. Society of Interventional Radiology Standards of Practice Committee. Quality improvement guidelines for the reporting and archiving of interventional radiology procedures. J Vasc Interv Radiol 2003; $14: 293-\$ 295$
3 Yadav JS, Sachar R, Casserly IP. Manual of peripheral vascular intervention. Philadelphia: Lippincott Williams \& Wilkens; 2005: $344-360$

4 Cardiovascular and Interventional Radiological Society of Europe (CIRSE). CIRSE IR Patient Safety Checklist. Im Internet: http://www.cirse.org/files/files/Profession/IR_Checklist_new. pdf (Stand: 23.10.2016)

5 Lee MJ, Fanelli F, Haage P et al. Patient safety in interventional radiology: a CIRSE IR checklist. Cardiovasc Intervent Radiol 2012; 35: $244-246$

6 Durack JC. The value proposition of structured reporting in interventional radiology. AJR Am J Roentgenol 2014; 203: $734-738$

7 Omary RA, Bettmann MA, Cardella JF et al. Quality improvement guidelines for the reporting and archiving of interventional radiology procedures. J Vasc Interv Radiol 2002; 13 : $879-881$

8 Collins N, Horlick E, Dzavik V. Triple wire technique for removal of fractured angioplasty guidewire. J Invasive Cardiol 2007; 19: E230-E234

9 Capuano F, Simon C, Roscitano A et al. Percutaneous transluminal coronary angioplasty hardware entrapment: guidewire entrapment. J Cardiovasc Med (Hagerstown) 2008; 9: 1140 1141

10 Ozkan M, Yokusoglu M, Uzun M. Retained percutaneous transluminal coronary angioplasty guidewire in coronary circulation. Acta Cardiol 2005; 60: 653-654

11 Martí V, Markarian L. Angioplasty guidewire entrapment affer stent implantation: report of two cases and review of the literature. Arch Cardiol Mex 2007; 77: 54- 57

12 Kang JH, Rha SW, Lee DI et al. Successful retrieval of a fractured and entrapped 0. 035-inch terumo wire in the femoral artery using biopsy forceps. Korean Circ J 2012; 42: 201 - 204

13 Rossi M, Citone M, Krokidis M et al. Percutaneous retrieval of a guide wire fragment with the use of an angioplasty balloon and an angiographic catheter: the sandwich technique. Cardiovasc Intervent Radiol 2013; 36: 1707 - 1710

14 Suri S, Nagarsheth KH, Goraya S et al. A novel technique to retrieve a maldeployed vascular closure device. J Endovasc Ther 2015; 22: $71-73$

15 Maxien D, Behrends B, Eberhardt KM et al. Endovascular treatment of acute limb ischemia caused by an intravascularly deployed bioabsorbable plug of a vascular closure device. Vasa 2013; 42: $144-148$

16 Cahill TJ, Choji K, Kardos A. Fluoroscopy-guided snare retrieval of the celt $A C D\left({ }^{\circledR}\right)$ metallic vascular closure device following failed deployment. Catheter Cardiovasc Interv 2014; 83: $556-559$

17 Kara K, Mahabadi AA, Rothe $\mathrm{H}$ et al. Safety and effectiveness of a novel vascular closure device: a prospective study of the ExoSeal compared to the Angio-Seal and ProGlide. J Endovasc Ther 2014; 21: $822-828$

18 Boersma D, van Strijen MJ, Kloppenburg GT et al. Endovascular retrieval of a dislodged femoral arterial closure device with Alligator forceps. J Vasc Surg 2012; 55: 1150-1152

19 Cikirikcioglu M, Cherian S, Keil V et al. Surgical treatment of complications associated with the Angio-Seal vascular closure device. Ann Vasc Surg 2011; 25: 557 
20 Schiele TM, Rademacher A, Meissner O et al. Acute limb ischemia after femoral arterial closure with a vascular sealing device: successful endovascular treatment. Vasa 2004; 33 : $252-256$

21 Durão C, Barros A, Guerreiro R et al. "Death by a thread" peritonitis due to visceral perforation by a guide wire, during proximal femur osteosynthesis with DHS: a fatal case and legal implications. Forensic Sci Int 2015; 249: e12-e14

22 Hiroshima Y, Tajima K, Shiono Y et al. Soft J-tipped guide wireinduced cardiac perforation in a patient with right ventricular lipomatosis and wall thinning. Intern Med 2012; 51: 26092612

23 Lee SY, Kim SM, Bae JW et al. Renal artery perforation related with hydrophilic guide wire during coronary intervention: successful treatment with polyvinyl alcohol injection. Can J Cardiol 2012; 28: 612.e5-7

24 Hong YM, Lee SR. A case of guide wire fracture with remnant filaments in the left anterior descending coronary artery and aorta. Korean Circ J 2010; 40: 475-477

25 Tanaka S, Nishigaki K, Ojio S et al. Transcatheter embolization by autologous blood clot is useful management for small side branch perforation due to percutaneous coronary intervention guide wire. J Cardiol 2008; 52: 285-289

26 Störger H, Ruef J. Closure of guide wire-induced coronary artery perforation with a two-component fibrin glue. Catheter Cardiovasc Interv 2007; 70: $237-240$
27 Axelrod DJ, Freeman H, Pukin L et al. Guide wire perforation leading to fatal perirenal hemorrhage from transcortical collaterals after renal artery stent placement. J Vasc Interv Radiol 2004; 15: 985 - 987

28 Blake PG, Uldall R. Cardiac perforation by a guide wire during subclavian catheter insertion. Int J Artif Organs 1989; 12: $111-113$

29 Taherioun M, Namazi MH, Safi M et al. Stent underexpansion in angiographic guided percutaneous coronary intervention, despite adjunctive balloon post-dilatation, in drug eluting stent era. ARYA Atheroscler 2014; 10: 13-17

30 Meadows J, Teitel D, Moore P. Anatomic and technical predictors of stent malposition during implantation for vascular obstruction in patients with congenital and acquired heart disease. JACC Cardiovasc Interv 2010; 3: 1080-1086

31 Broadbent LP, Moran CJ, Cross DT3rd et al. Management of neuroform stent dislodgement and misplacement. AJNR Am J Neuroradiol 2003; 24: 1819-1822

32 Dawson DL, Terramani TT, Loberman Z et al. Simple technique to ensure coaxial guidewire positioning for placement of iliac limb of modular aortic endograft. J Interv Cardiol 2003; 16 : $223-226$

33 Cho YJ, Han SS, Lee SC. Guidewire malposition during central venous catheterization despite the use of ultrasound guidance. Korean J Anesthesiol 2013; 64: 469-471 


\section{CME-Fragen}

\section{CME.thieme.de}

\section{CME-Teilnahme}

- Viel Erfolg bei Ihrer CME-Teilnahme unter http://cme.thieme.de

- Diese Fortbildungseinheit ist 12 Monate online für eine CME-Teilnahme verfügbar.

- Sollten Sie Fragen zur Online-Teilnahme haben, unter http://cme.thieme.de/hilfe finden Sie eine ausführliche Anleitung.

\section{1}

Welche der nachfolgenden Aussagen zur Einteilung von Komplikationen ist richtig?
A Minorkomplikationen führen zu einer Verlängerung des Krankenhausaufenthalts auf mehr als $48 \mathrm{~h}$.

B Eine Majorkomplikation erfordert immer eine weitere Behandlung.

C Das nicht behandlungswürdige Leistenhämatom ist eine Majorkomplikation.

D Minorkomplikationen erfordern immer eine weitere interventionelle Therapie.

E Eine Majorkomplikation führt in der Regel zum Ableben des Patienten.

\section{2}

Welcher der folgenden Faktoren hat den geringsten Einfluss auf die Rate an Komplikationen bei vaskulären Eingriffen?
A Compliance des Patienten
B Versichertenstatus des Patienten
C Vorerkrankungen des Patienten
D Erfahrung des Interventionalisten
E Komplexität des Eingriffs

\section{3}

Welche der folgenden Komplikationen treten typischerweise am Punktionsort auf?
A distale Embolisation
B Non-Target-Embolisation
C Postimplantationssyndrom
D Blutung, Hämatom, Aneurysma spurium, Gefäßverschluss
E Dissektion, Perforation, Stent-Verschluss

Wodurch lassen sich Verwechslungskomplikationen vermeiden?
A Implementierung von SOP und Gebrauch von Checklisten
B autogenes Training
C elektronische Patientenanmeldung
D Prämedikation
E Sichtung vorhandener Voraufnahmen

Welche Aussage zur Leistenarterienpunktion trifft nicht zu?
A Verschluss durch Kollagenpfropfen nach Anwendung eines vaskulären Verschlusssystems
B Dissektion, Perforation, Reverschluss, arteriovenöse Fistel
C Aneurysma spurium
D anaphylaktischer Schock
E Infektion

A Die unkontrollierte Manipulation eines hydrophil beschichteten Drahtes in Kombination mit einer scharfen Hohlnadel kann zur Abschälung der Beschichtung führen.

B Intravasale Fremdkörper können zu einem arteriellen Verschluss führen.

C Sollte es beim Zurückziehen eines hydrophil beschichteten Drahtes durch eine geschliffene Hohlnadel zu einem Widerstand kommen, sollte der Draht einschließlich der Punktionsnadel entfernt werden.

D Bei Verwendung einer asymmetrisch geschliffenen Hohlnadel sollten bevorzugt hydrophil beschichtete Führungsdrähte zur Anwendung kommen.

E latrogene intravasale Fremdkörper sollten, wenn möglich, interventionell geborgen werden. 


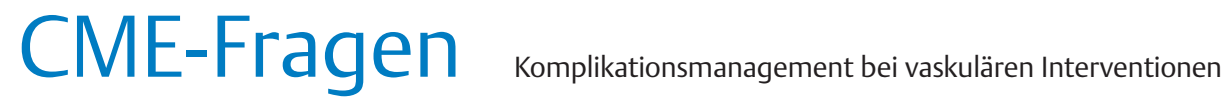

\section{7}

Nach Anwendung eines arteriellen Verschlusssystems kommt es zur Ablösung eines Kollagenpfropfens in die A. femoralis. Welche der folgenden Maßnahmen stellt keine mögliche Strategie zur Komplikationsbehandlung dar?
A intraarterielle Lysetherapie
B PTA- bzw. Stent-Implantation
C Bergung durch Schlingen- oder Aspirationskatheter
D offen-chirurgische Freilegung und Entfernung
E Atherektomie mit Filterprotektion

\section{8}

Welche Aussage zur Seitenastperforation durch hydrophil beschichtete Führungsdrähte trifft zu?

A Die hydrophile Beschichtung von Führungsdrähten verhindert zuverlässig die Verletzung kleinerer Seitenäste.

B Die visuelle Kontrolle der distalen Führungsdrahtspitze während des Vorschiebens von Kathetern und anderen Interventionsmaterialien ist geeignet, eine Verletzung von Seitenästen zu vermeiden.

C Ein durch den Patienten während der Intervention geäußertes plötzliches Schmerzereignis außerhalb des Behandlungsorts ist zumeist nur Ausdruck der allgemeinen psychischen Anspannung.

D Bei Seitenastperforation sollte die betroffene Arterie operativ ligiert werden.

E Eine Seitenastperforation ist für den Patienten immer ohne klinische Konsequenz.

\section{9}

Welche der folgenden Aussagen zur Stent-Migration trifft nicht zu?
A Stent-Ballonkatheter sollten kontrolliert und vorsichtig unter Durchleuchtung vorgeschoben werden.

B Kann ein ballonexpandierbarer Stent eine Läsion nicht passieren, sollte eine Vordilatation erfolgen.

C Ein Stent- bzw. Ballonkatheter sollte nie unkontrolliert und gegen einen Widerstand vor- oder zurückgeschoben werden.

D Nach einer Stent-Migration ist die Bergung mit einem Schlingenkatheter eine mögliche Option.

E Industriell vormontierte ballonexpandierbare Stents lösen sich nicht vom Ballon ab und können daher bedenkenlos auch ohne Vordilatation oder Schleusenschutz eingesetzt werden.

\section{0}

Welche der folgenden Maßnahmen trägt nicht dazu bei, eine Stent-Fehlplatzierung zu vermeiden?
A Sicherung der intraluminalen Lage des Führungsdrahts im Zielgefäß durch Angiografie in mindestens 2 Ebenen

B Orientierung an anatomischen Landmarken

C Fluoroskopie mit erhöhter Pulsrate und Vergrößerung während der Freisetzung

D Visualisierung der intraluminalen Katheterlage distal der Läsion durch Kontrastmittelgabe

E Gabe von Thrombozytenaggregationshemmern 